International Journal of Pure and Applied Mathematics

Volume 107 No. 4 2016, 983-995

ISSN: 1311-8080 (printed version); ISSN: 1314-3395 (on-line version)

url: http://www.ijpam.eu

doi: 10.12732/ijpam.v107i4.16

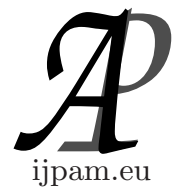

\title{
MULTIPLE VALUES AND UNIQUENESS OF MEROMORPHIC FUNCTIONS ON ANNULI
}

\author{
Renukadevi S. Dyavanal ${ }^{1} \S$, Ashok Rathod ${ }^{2}$ \\ ${ }^{1,2}$ Department of Mathematics \\ Karnatak University \\ Dharwad, 580 003, INDIA
}

\begin{abstract}
The purpose of this paper is to deal with the multiple values and deficiencies on the uniqueness problem of meromorphic functions on annuli, and extend some uniqueness theorems of meromorphic functions dealing with multiple values and deficient values to meromorphic functions on annuli.
\end{abstract}

AMS Subject Classification: 30D35

Key Words: Nevanlinna theory, the annulus

\section{Introduction}

We assume that the reader is familiar with Nevanlinna's theory of meromorphic functions (see [13]). The uniqueness of meromorphic functions in the complex plane $\mathbb{C}$ is an important subject in the value distribution theory. The uniqueness of meromorphic functions with shared values on $\mathbb{C}$ attracted many investigations (see book [13]). Here we shall mainly study the uniqueness of meromorphic functions in doubly connected domains of complex plane $\mathbb{C}$. By Doubly connected mapping theorem [9] each doubly connected domain is conformally equivalent to the annulus $\{z: r<|z|<R\}, 0 \leq r<R \leq+\infty$. We consider only two cases : $r=0, R=+\infty$ simultaneously and $0 \leq r<R \leq+\infty$. In the latter case the homothety $z \mapsto \frac{z}{r R}$ reduces the given domain to the annulus $\left\{z: \frac{1}{R_{0}}<|z|<R_{0}\right\}$, where $R_{0}=\sqrt{\frac{R}{r}}$. Thus, in two cases every annulus is invariant with respect to the inversion $z \mapsto \frac{1}{z}$.

Received: January 12, 2016

Published: May 7, 2016

${ }^{\S}$ Correspondence author (c) 2016 Academic Publications, Ltd.

url: www.acadpubl.eu 
The uniqueness theory of meromorphic function is an interesting problem, recently Khrystiyanyn and Kondratyuk [6, 7 ] proposed the Nevenlinna theory of meromorphic functions on annuli, see also an important paper [3]. We will show the basic notions of the Nevanlinna theory on annuli in the next section. In this paper, we mainly study the uniqueness problem of meromorphic functions on annuli, and extend some uniqueness theorems of meromorphic functions dealing with multiple values and deficient values to meromorphic functions on annili.

\section{Basic Notations in the Nevanlinna Theory on Annuli}

Let $f$ be a meromorphic function on the annulus $\mathbb{A}=\left\{z: \frac{1}{R_{0}}<|z|<R_{0}\right\}$. We recall classical notations of Nevanlinna theory as follows

$$
\begin{aligned}
& N(R, f)=\int_{0}^{R} \frac{n(t, f)-n(0, f)}{t} d t+n(0, f) \log R, \\
& m(R, f)=\frac{1}{2 \pi} \int_{0}^{2 \pi} \log ^{+}\left|f\left(R e^{i \theta}\right)\right| d \theta, \quad T(R, f)=N(R, f)+m(R, f),
\end{aligned}
$$

where $\log ^{+} x=\max \{\log x, 0\}$, and $n(t, f)$ is the counting function of poles of the function $f$ in $\{z:|z| \leq t\}$. Here we show the notations of the Nevanlinna theory on annuli. Let

$$
\begin{array}{cc}
N_{1}(R, f)=\int_{\frac{1}{R}}^{1} \frac{n_{1}(t, f)}{t} d t, & N_{2}(R, f)=\int_{1}^{R} \frac{n_{2}(t, f)}{t} d t, \\
m_{0}(R, f)=m(R, f)+m\left(\frac{1}{R}, f\right), & N_{0}(R, f)=N_{1}(R, f)+N_{2}(R, f),
\end{array}
$$

where $n_{1}(t, f)$ and $n_{2}(t, f)$ are the counting functions of the poles of the function $f$ in $\{z: t<|z| \leq 1\}$ and $\{z: 1<|z| \leq t\}$, respectively. The Nevanlinna charecteristic of $f$ on the annulus $\mathbb{A}$ is defined by

$$
T_{0}(R, f)=m_{0}(r, f)+N_{0}(R, f)-2 m(1, f) .
$$

Definition - [4] Let $f(z)$ be a non-constant meromorphic function on the annulus $\mathbb{A}\left(R_{0}\right)=\left\{z: 1 / R_{0}<|z|<R_{0}\right\}$, where $1<R_{0}<+\infty$. The function $f$ is called a transcedental or admissible meromorphic function on the annulus $\mathbb{A}\left(R_{0}\right)$ provided that

$$
\lim _{R \rightarrow \infty} \sup \frac{T_{0}(R, f)}{\log R}=\infty, \quad 1<R<R_{0}=+\infty
$$


or

$$
\lim _{R \rightarrow R_{0}} \sup \frac{T_{0}(R, f)}{-\log \left(R_{0}-R\right)}=\infty, \quad 1<R<R_{0}<+\infty
$$

respectively.

Thus for a transcedental or admissible meromorphic function on the annulus $\mathbb{A}, S(R, f)=o\left(T_{0}(R, f)\right)$ holds for all $1<R<R_{0}$ except for the set $\triangle_{R}$ or the set $\triangle_{R}^{\prime}$ mentioned in Theorem 2.1, respectively.

Definition - Let $f(z)$ be a meromorphic functions on the annulus $\mathbb{A}=$ $\left\{z: \frac{1}{R_{0}}<|z|<R_{0}\right\}$, where $1<R<R_{0} \leq+\infty$. Let $a$ be any arbitrary complex number. The Valiron deficiency of $f(z)$ on the annulus $\mathbb{A}$ with respect to the value ' $a$ ' will be defined by

$$
\Delta_{0}(a, f)=\varlimsup_{r \rightarrow \infty} \frac{m_{0}\left(R, \frac{1}{f-a}\right)}{T_{0}(R, f)}=1-\varliminf_{r \rightarrow \infty} \frac{N_{0}\left(R, \frac{1}{f-a}\right)}{T_{0}(R, f)} .
$$

We denote the deficiency of $a \in \overline{\mathbb{C}}=\mathbb{C} \cup\{\infty\}$ with respect to a meromorphic function $f$ on the annulus $\mathbb{A}$ by

$$
\delta_{0}(a, f)=\delta_{0}(0, f-a)=\varliminf_{r \rightarrow R_{0}} \frac{m_{0}\left(R, \frac{1}{f-a}\right)}{T_{0}(R, f)}=1-\varlimsup_{r \rightarrow R_{0}} \frac{N_{0}\left(R, \frac{1}{f-a}\right)}{T_{0}(R, f)},
$$

and denote the reduced deficiency by

$$
\Theta_{0}(a, f)=\Theta_{0}(0, f-a)=1-\varlimsup_{r \rightarrow R_{0}} \frac{\overline{N_{0}}\left(R, \frac{1}{f-a}\right)}{T_{0}(R, f)}
$$

where

$$
\begin{aligned}
\overline{N_{0}}\left(R, \frac{1}{f-a}\right) & =\overline{N_{1}}\left(R, \frac{1}{f-a}\right)+\overline{N_{2}}\left(R, \frac{1}{f-a}\right) \\
& =\int_{\frac{1}{R}}^{1} \frac{\overline{n_{1}}\left(t, \frac{1}{f-a}\right)}{t} d t+\int_{1}^{R} \frac{\overline{n_{2}}\left(t, \frac{1}{f-a}\right)}{t} d t
\end{aligned}
$$

in which each zero of the function $f-a$ is counted only once.

We use $\bar{n}_{1}^{k)}\left(t, \frac{1}{f-a}\right)$ (or $\left.\bar{n}_{1}^{(k}\left(t, \frac{1}{f-a}\right)\right)$ to denote the counting function of poles of the function $\frac{1}{f-a}$ with the multiplicities $\leq k \quad$ or $>k$ ) in $\{z: t<$ $|z| \leq 1\}$, each point counted only once. Similarly, we can give the notations \left.${\overline{N_{1}}}^{k)}(t, f),{\overline{N_{1}}}^{(k}(t, f),{\overline{N_{2}}}^{k)}(t, f),{\overline{N_{2}}}^{(k}(t, f),{\overline{N_{0}}}^{k}\right)(t, f),{\overline{N_{0}}}^{(k}(t, f)$. 
Lemma 2.1. $[3]$ Let $f$ be a non constant meromorphic function on the annulus $\mathbb{A}=\left\{z: \frac{1}{R_{0}}<|z|<R_{0}\right\}$, where $1<R<R_{0} \leq+\infty$. Let ' $a$ ' be an arbitrary complex number, and $k$ be a positive integer. Then

(i) $\overline{N_{0}}\left(R, \frac{1}{f-a}\right) \leq \frac{k}{k+1}{\overline{N_{0}}}^{k)}\left(R, \frac{1}{f-a}\right)+\frac{1}{k+1} \overline{N_{0}}\left(R, \frac{1}{f-a}\right)$,

(ii) $\overline{N_{0}}\left(R, \frac{1}{f-a}\right) \leq \frac{k}{k+1}{\overline{N_{0}}}^{k)}\left(R, \frac{1}{f-a}\right)+\frac{1}{k+1} T_{0}(R, f)+O(1)$.

Theorem 2.1. $[3]$ (The Second Fundamental Theorem in the annulus). Let $f$ be a non constant meromorphic function on the annulus

$$
\mathbb{A}=\left\{z: \frac{1}{R_{0}}<|z|<R_{0}\right\},
$$

where $1<R<R_{0} \leq+\infty$. Let $a_{1}, a_{2}, \ldots, a_{q}$ be $q$ distinct complex numbers in the extended complex plane $\overline{\mathbb{C}}=\mathbb{C} \cup\{\infty\}$, let $k_{1}, k_{2}, \ldots, k_{q}$ be $q$ positive integers, and let $\lambda \geq 0$. Then:

(i) $(q-2) T_{0}(R, f)<\sum_{j=1}^{q} N_{0}\left(R, \frac{1}{f-a_{j}}\right)-N_{0}^{(1)}(R, f)+S(R, f)$,

(ii) $(q-2) T_{0}(R, f)<\sum_{j=1}^{q} \overline{N_{0}}\left(R, \frac{1}{f-a_{j}}\right)+S(R, f)$,

$$
\begin{aligned}
\text { (iii) }(q-2) T_{0}(R, f)< & \left.\sum_{j=1}^{q} \frac{k_{j}}{k_{j}+1}{\overline{N_{0}}}^{k_{j}}\right)\left(R, \frac{1}{f-a_{j}}\right) \\
& +\sum_{j=1}^{q} \frac{1}{k_{j}+1} N_{0}\left(R, \frac{1}{f-a_{j}}\right)+S(R, f),
\end{aligned}
$$

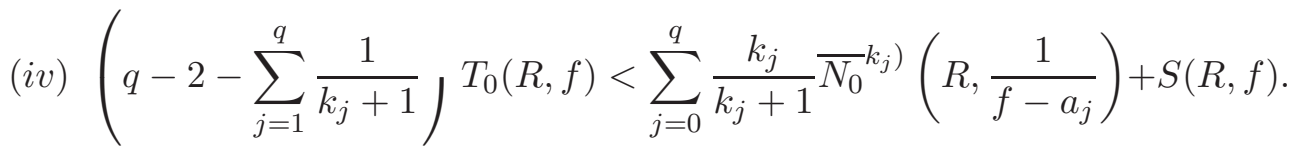

where

$$
N_{0}^{(1)}(R, f)=N_{0}\left(R, \frac{1}{f^{\prime}}\right)+2 N_{0}(R, f)-N_{0}\left(R, f^{\prime}\right)
$$

and 
1. In the case, $R_{0}=+\infty$,

$$
S(R, f)=O\left(\log \left(R T_{0}(R, f)\right)\right)
$$

for $R \in(1,+\infty)$ except for the set $\triangle_{R}$ such that $\int_{\triangle_{R}} R^{\alpha-1} d R<+\infty$;

2. In the case, $R_{0}<+\infty$,

$$
S(R, f)=O\left(\log \left(\frac{T_{0}(R, f)}{R_{0}-R}\right)\right)
$$

for $R \in\left(1, R_{0}\right)$ except for the set $\triangle_{R}^{\prime}$ such that $\int_{\triangle_{R}^{\prime}} \frac{d R}{\left(R_{0}-R^{\alpha-1}\right)}<+\infty$.

\section{Multiple Values and Uniqueness of Meromorphic Functions on Annuli}

Let $f$ be a meromorphic function on the annulus $\mathbb{A}=\left\{z: \frac{1}{R_{0}}<|z|<R_{0}\right\}$, where $1<R<R_{0} \leq+\infty$, and ' $a$ ' be a complex number in the extende complex plane $\overline{\mathbb{C}}=\mathbb{C} \cup\{\infty\}$. Write $E(a, f)=\{z \in \mathbb{A}: f(z)-a=0\}$, where each zero with multiplicity $m$ is counted $m$ times. If we ignore the multiplicity, then the set is denoted by $\bar{E}(a, f)$. We use $\bar{E}_{k)}(a, f)$ to denote the set of zeros of $f-a$ with multiplicities no greater than $k$, in which each zero is counted only once.

We now show our main results below which is an analog of a result on the plane $\mathbb{C}$ obtained by H. X. Yi [12](see Theorem 3.19 and 3.20 in [13]).

Theorem 3.1. Let $f_{1}$ and $f_{2}$ be two transcedental or admissible meromorphic functions on the annulus $\mathbb{A}=\left\{z: \frac{1}{R_{0}}<|z|<R_{0}\right\}$, where $1<R<$ $R_{0} \leq+\infty$. Let $a_{j}(j=1,2, \ldots, q)$ be $q$ distinct complex numbers in $\overline{\mathbb{C}}$, and $k_{j}(j=1,2, \ldots, q)$ be positive integers or $\infty$ satisfying

$$
k_{1} \geq k_{2} \geq \ldots \geq k_{q}
$$

and

$$
\bar{E}_{\left.k_{j}\right)}\left(a_{j}, f_{1}\right)=\bar{E}_{\left.k_{j}\right)}\left(a_{j}, f_{2}\right), \quad(j=1,2, . ., q) .
$$

Set

$$
A_{i}=\frac{\delta_{0}\left(a_{1}, f_{i}\right)+\delta_{0}\left(a_{2}, f_{i}\right)}{k_{3}+1}+\sum_{j=3}^{q} \frac{\delta_{0}\left(a_{j}, f_{i}\right)}{k_{j}+1} \quad(i=1,2) .
$$


If

$$
\min \left\{A_{1}, A_{2}\right\} \geq 2-\sum_{j=3}^{q} \frac{k_{j}}{k_{j}+1}
$$

and

$$
\max \left\{A_{1}, A_{2}\right\}>2-\sum_{j=3}^{q} \frac{k_{j}}{k_{j}+1},
$$

then $f_{1}(z) \equiv f_{2}(z)$.

Proof. We may assume, without loss of generality, that all $a_{j}(j=1,2, \ldots, q)$ are finite, otherwise, a suitable Mobius transformation will be done. From Theorem 2.1, we have

$$
\begin{aligned}
\left.(q-2) T_{0}\left(R, f_{1}\right)<\sum_{j=1}^{q} \frac{k_{j}}{k_{j}+1} \bar{N}_{0} k_{j}\right) & \left(R, \frac{1}{f_{1}-a_{j}}\right) \\
& +\sum_{j=1}^{q} \frac{1}{k_{j}+1} N_{0}\left(R, \frac{1}{f_{1}-a_{j}}\right)+S\left(R, f_{1}\right) .
\end{aligned}
$$

From (3.1) and (3.5), we have

$$
\begin{aligned}
(q-2) T_{0}\left(R, f_{1}\right)< & \left.\frac{k_{3}}{k_{3}+1} \sum_{j=1}^{q} \overline{N_{0}} k_{j}\right)\left(R, \frac{1}{f_{1}-a_{j}}\right) \\
& +\sum_{j=1}^{2}\left(\frac{k_{j}}{k_{j}+1}-\frac{k_{3}}{k_{3}+1}\right) N_{0}\left(R, \frac{1}{f_{1}-a_{j}}\right) \\
& +\sum_{j=1}^{q} \frac{1}{k_{j}+1} N_{0}\left(R, \frac{1}{f_{1}-a_{j}}\right)+S\left(R, f_{1}\right) \\
= & \left.\frac{k_{3}}{k_{3}+1} \sum_{j=1}^{q} \frac{1}{N_{0}} k_{j}\right)\left(R, \frac{1}{f_{1}-a_{j}}\right) \\
& +\sum_{j=1}^{2}\left(\frac{k_{j}}{k_{j}+1}-\frac{k_{3}}{k_{3}+1}\right)\left(1-\delta_{0}\left(a_{j}, f_{1}\right)\right) T_{0}\left(R, f_{1}\right) \\
& +\sum_{j=1}^{q} \frac{1}{k_{j}+1}\left(1-\delta_{0}\left(a_{j}, f_{1}\right)\right) T_{0}\left(R, f_{1}\right)+S\left(R, f_{1}\right) \\
= & \left.\frac{k_{3}}{k_{3}+1} \sum_{j=1}^{q} \frac{1}{N_{0}} k_{j}\right)\left(R, \frac{1}{f_{1}-a_{j}}\right)
\end{aligned}
$$




$$
\begin{aligned}
& +\sum_{j=1}^{2}\left(\frac{k_{j}}{k_{j}+1}+\frac{1}{k_{j}+1}\right)\left(1-\delta_{0}\left(a_{j}, f_{1}\right)\right) T_{0}\left(R, f_{1}\right) \\
& -\sum_{j=1}^{2} \frac{k_{3}}{k_{3}+1}\left(1-\delta_{0}\left(a_{j}, f_{1}\right)\right) T_{0}\left(R, f_{1}\right) \\
& +\sum_{j=3}^{q} \frac{1}{k_{j}+1}\left(1-\delta_{0}\left(a_{j}, f_{1}\right)\right) T_{0}\left(R, f_{1}\right)+S\left(R, f_{1}\right) \\
= & \left.\frac{k_{3}}{k_{3}+1} \sum_{j=1}^{q} \frac{1}{N_{0}} k_{j}\right)\left(R, \frac{1}{f_{1}-a_{j}}\right)+2 T_{0}\left(R, f_{1}\right) \\
& -\sum_{j=1}^{2} \delta_{0}\left(a_{j}, f_{1}\right) T_{0}\left(R, f_{1}\right)-\frac{2 k_{3}}{k_{3}+1} T_{0}\left(R, f_{1}\right) \\
& +\sum_{j=1}^{2} \frac{k_{3}}{k_{3}+1} \delta_{0}\left(a_{j}, f_{1}\right) T_{0}\left(R, f_{1}\right) \\
& -\sum_{j=3}^{q} \frac{1}{k_{j}+1}\left(\delta_{0}\left(a_{j}, f_{1}\right)\right) T_{0}\left(R, f_{1}\right)+S\left(R, f_{1}\right) \\
& +\sum_{j=3} \frac{1}{k_{3}+1}\left(1-\delta_{0}\left(a_{j}, f_{1}\right)\right) T_{0}\left(R, f_{1}\right)+S\left(R, f_{1}\right) \\
& -\sum_{j=1}^{2} \frac{\delta_{0}\left(a_{j}, f_{1}\right)}{k_{3}+1} T_{0}\left(R, f_{1}\right) \\
& \left.+k_{3} k_{j}\right)\left(R, \frac{1}{f_{1}-a_{j}}\right)+2 T_{0}\left(R, f_{1}\right)
\end{aligned}
$$

Therefore,

$$
\begin{gathered}
\left(\frac{\delta_{0}\left(a_{1}, f_{1}\right)+\delta_{0}\left(a_{2}, f_{1}\right)}{k_{3}+1}+\sum_{j=3}^{q} \frac{\delta_{0}\left(a_{j}, f_{1}\right)}{k_{j}+1}+\frac{2 k_{3}}{k_{3}+1}+\sum_{j=3}^{q} \frac{k_{j}}{k_{j}+1}-2 T_{0}\left(R, f_{1}\right)\right. \\
\left.<\frac{k_{3}}{k_{3}+1} \sum_{j=1}^{q} \overline{N_{0}} k_{j}\right)\left(R, \frac{1}{f_{1}-a_{j}}\right)
\end{gathered}
$$




$$
\begin{aligned}
+S\left(R, f_{1}\right)\left(A_{1}\right. & +\frac{2 k_{3}}{k_{3}+1}+\sum_{j=3}^{q} \frac{k_{j}}{k_{j}+1}-2 T_{0}\left(R, f_{1}\right) \\
& \left.<\frac{k_{3}}{k_{3}+1} \sum_{j=1}^{q}{\overline{N_{0}}}^{k_{j}}\right)\left(R, \frac{1}{f_{1}-a_{j}}\right)+S\left(R, f_{1}\right) .
\end{aligned}
$$

If $f_{1}(z) \not \equiv f_{2}(z)$, then from (3.2), we have

$$
\left.\sum_{j=1}^{q}{\overline{N_{0}}}^{k_{j}}\right)\left(R, \frac{1}{f_{1}-a_{j}}\right) \leq N_{0}\left(R, \frac{1}{f_{1}-f_{2}}\right) \leq T_{0}\left(R, f_{1}\right)+T_{0}\left(R, f_{2}\right)+O(1) .
$$

From (3.7) and (3.8), we get

$$
\left(A_{1}+\frac{k_{3}}{k_{3}+1}+\sum_{j=3}^{q} \frac{k_{j}}{k_{j}+1}-2\right)_{0}\left(R, f_{1}\right)<\frac{k_{3}}{k_{3}+1} T_{0}\left(R, f_{2}\right)+S\left(R, f_{1}\right)
$$

Combining (3.3) and (3.9)

$$
T_{0}\left(R, f_{1}\right)<T_{0}\left(R, f_{2}\right)+S\left(R, f_{1}\right) .
$$

Similarly,

$$
\left(A_{2}+\frac{2 k_{3}}{k_{3}+1}+\sum_{j=3}^{q} \frac{k_{j}}{k_{j}+1}-2\right)^{T_{0}}\left(R, f_{2}\right)<\frac{k_{3}}{k_{3}+1} T_{0}\left(R, f_{1}\right)+S\left(R, f_{2}\right)
$$

and

$$
T_{0}\left(R, f_{2}\right)<T_{0}\left(R, f_{1}\right)+S\left(R, f_{2}\right) .
$$

From (3.9) and (3.12) we have

$$
\left(A_{1}+\sum_{j=3}^{q} \frac{k_{j}}{k_{j}+1}-2, T_{0}\left(R, f_{1}\right)<S\left(R, f_{1}\right)\right.
$$

while (3.10) and (3.11) mean

$$
\left(A_{2}+\sum_{j=3}^{q} \frac{k_{j}}{k_{j}+1}-2, T_{0}\left(R, f_{2}\right)<S\left(R, f_{2}\right)\right.
$$

However according to (3.4), we know that one of the inequalities (3.13), (3.14) is a contradiction.

Hence $f_{1}(z) \equiv f_{2}(z)$. 
As a consequence of Theorem 3.1, we get the following corollary

Corollary 3.1. Let $f_{1}$ and $f_{2}$ be two transcedental or admissible meromorphic functions on the annulus $\mathbb{A}=\left\{z: \frac{1}{R_{0}}<|z|<R_{0}\right\}$, where $1<R<$ $R_{0} \leq+\infty$. Let $a_{j}(j=1,2, \ldots, q)$ be $q$ distinct complex numbers. Then:

(i) If $q=6$ and $\bar{E}_{1)}\left(a_{j}, f_{1}\right)=\bar{E}_{1)}\left(a_{j}, f_{2}\right),(j=1,2, . ., 6)$,

$$
\sum_{j=1}^{6} \max \left\{\delta_{0}\left(a_{j}, f_{1}\right), \delta_{0}\left(a_{j}, f_{2}\right)\right\}>0
$$

then $f_{1}(z) \equiv f_{2}(z)$;

(ii) If $q=5$ and $\bar{E}_{2)}\left(a_{j}, f_{1}\right)=\bar{E}_{2)}\left(a_{j}, f_{2}\right),(j=1,2, . ., 5)$,

$$
\sum_{j=1}^{5} \max \left\{\delta_{0}\left(a_{j}, f_{1}\right), \delta_{0}\left(a_{j}, f_{2}\right)\right\}>0
$$

then $f_{1}(z) \equiv f_{2}(z)$;

(iii) If $q=5$ and $\bar{E}_{1)}\left(a_{j}, f_{1}\right)=\bar{E}_{1)}\left(a_{j}, f_{2}\right),(j=1,2, . ., 5)$,

$$
\sum_{j=1}^{5} \min \left\{\delta_{0}\left(a_{j}, f_{1}\right), \delta_{0}\left(a_{j}, f_{2}\right)\right\} \geq 1
$$

$\max \left\{\delta_{0}\left(a_{j}, f_{1}\right), \delta_{0}\left(a_{j}, f_{2}\right)\right\}>1$, then $f_{1}(z) \equiv f_{2}(z)$.

Theorem 3.2. Let $f_{1}$ and $f_{2}$ be two transcedental or admissible meromorphic functions on the annulus $\mathbb{A}=\left\{z: \frac{1}{R_{0}}<|z|<R_{0}\right\}$, where $1<R<$ $R_{0} \leq+\infty$. Let $a_{j}(j=1,2, \ldots, q)$ be $q$ distinct complex numbers in $\overline{\mathbb{C}}$, and $k_{j}(j=1,2, \ldots, q)$ be positive integers or $\infty$ satisfying (3.1) and (3.2). Set

$$
B_{i}=\frac{\Delta_{0}\left(a_{1}, f_{i}\right)+\Delta_{0}\left(a_{2}, f_{i}\right)}{k_{3}+1}+\sum_{j=1}^{q} \frac{\Delta_{0}\left(a_{j}, f_{i}\right)}{k_{j}+1} \quad(i=1,2) .
$$

If

$$
\sum_{j=3}^{q} \frac{k_{j}}{k_{j}+1}=2
$$

and

$$
\max \left\{B_{1}, B_{2}\right\}>0
$$

Then $f_{1}(z) \equiv f_{2}(z)$. 
Proof. We may assume, without loss of generality, that all $a_{j}(j=1,2, \ldots, q)$ are finite, otherwise, a suitable Mobius transformation will be done. From (3.6), we have

$$
\begin{aligned}
& (q-2) T_{0}\left(R, f_{1}\right) \\
& \left.<\frac{k_{3}}{k_{3}+1} \sum_{j=1}^{q} \overline{N_{0}} k_{j}\right)\left(R, \frac{1}{f_{1}-a_{j}}\right)+\sum_{j=1}^{2}\left(\frac{k_{j}}{k_{j}+1}-\frac{k_{3}}{k_{3}+1}\right) N_{0}\left(R, \frac{1}{f_{1}-a_{j}}\right) \\
& +\sum_{j=1}^{q} \frac{1}{k_{j}+1} N_{0}\left(R, \frac{1}{f_{1}-a_{j}}\right)+S\left(R, f_{1}\right) \\
& \left.=\frac{k_{3}}{k_{3}+1} \sum_{j=1}^{q} \overline{N_{0}} k_{j}\right)\left(R, \frac{1}{f_{1}-a_{j}}\right)+\sum_{j=1}^{2} N_{0}\left(R, \frac{1}{f_{1}-a_{j}}\right) \\
& -\sum_{j=1}^{2} \frac{k_{3}}{k_{3}+1} N_{0}\left(R, \frac{1}{f_{1}-a_{j}}\right)+\sum_{j=3}^{q} \frac{1}{k_{j}+1} N_{0}\left(R, \frac{1}{f_{1}-a_{j}}\right)+S\left(R, f_{1}\right) \\
& =\frac{k_{3}}{k_{3}+1} \sum_{j=1}^{q} N_{0} k_{j}\left(R, \frac{1}{f_{1}-a_{j}}\right)+\sum_{j=1}^{2}\left(1-\frac{k_{3}}{k_{3}+1}\right) N_{0}\left(R, \frac{1}{f_{1}-a_{j}}\right) \\
& +\sum_{j=3}^{q} \frac{1}{k_{j}+1} N_{0}\left(R, \frac{1}{f_{1}-a_{j}}\right)+S\left(R, f_{1}\right)
\end{aligned}
$$

Therefore,

$$
\begin{aligned}
(q-2) T_{0}\left(R, f_{1}\right)< & \left.\frac{k_{3}}{k_{3}+1} \sum_{j=1}^{q} \overline{N_{0}} k_{j}\right)\left(R, \frac{1}{f_{1}-a_{j}}\right) \\
& +\frac{1}{k_{3}+1} \sum_{j=1}^{2} N_{0}\left(R, \frac{1}{f_{1}-a_{j}}\right) \\
& +\sum_{j=3}^{q} \frac{1}{k_{j}+1} N_{0}\left(R, \frac{1}{f_{1}-a_{j}}\right)+S\left(R, f_{1}\right)
\end{aligned}
$$

If $f_{1}(z) \not \equiv f_{2}(z)$, then from $(3.2)$, we have

$$
\begin{aligned}
\left.\sum_{j=1}^{q}{\overline{N_{0}}}^{k_{j}}\right)\left(R, \frac{1}{f_{1}-a_{j}}\right) \leq N_{0}\left(R, \frac{1}{f_{1}-f_{2}}\right) \\
\quad \leq T_{0}\left(R, f_{1}\right)+T_{0}\left(R, f_{2}\right)+O(1) .
\end{aligned}
$$


From (3.17) and (3.18), we get

$$
\begin{aligned}
(q-2) T_{0}\left(R, f_{1}\right)< & \frac{k_{3}}{k_{3}+1}\left(T_{0}\left(R, f_{1}\right)+T_{0}\left(R, f_{2}\right)\right) \\
& +\frac{1}{k_{3}+1} \sum_{j=1}^{2} N_{0}\left(R, \frac{1}{f_{1}-a_{j}}\right) \\
& +\sum_{j=3}^{q} \frac{1}{k_{j}+1} N_{0}\left(R, \frac{1}{f_{1}-a_{j}}\right)+S\left(R, f_{1}\right)
\end{aligned}
$$

Similarly,

$$
\begin{aligned}
(q-2) T_{0}\left(R, f_{2}\right)< & \frac{k_{3}}{k_{3}+1}\left(T_{0}\left(R, f_{1}\right)+T_{0}\left(R, f_{2}\right)\right) \\
& +\frac{1}{k_{3}+1} \sum_{j=1}^{2} N_{0}\left(R, \frac{1}{f_{2}-a_{j}}\right) \\
& +\sum_{j=3}^{q} \frac{1}{k_{j}+1} N_{0}\left(R, \frac{1}{f_{2}-a_{j}}\right)+S\left(R, f_{2}\right)
\end{aligned}
$$

From (3.19) and (3.20), we get

$$
\begin{aligned}
\left(q-2-\frac{2 k_{3}}{k_{3}+1}\right) \sum_{i=1}^{2} T_{0}\left(R, f_{i}\right) & <\frac{1}{k_{3}+1} \sum_{i=1}^{2} \sum_{j=1}^{2} N_{0}\left(R, \frac{1}{f_{i}-a_{j}}\right) \\
& +\sum_{i=1}^{2} \sum_{j=3}^{q} \frac{1}{k_{j}+1} N_{0}\left(R, \frac{1}{f_{i}-a_{j}}\right) \\
& +S\left(R, f_{1}\right)+S\left(R, f_{2}\right) \\
& <\left(\frac{2}{k_{3}+1}+\sum_{j=3}^{q} \frac{1}{k_{j}+1}+O(1) \sum_{i=1}^{2} T_{0}\left(R, f_{i}\right) .\right.
\end{aligned}
$$

From (3.15) and (3.21), we get

$$
\left(q-2-\frac{2 k_{3}}{k_{3}+1}\right)=\left(\frac{2}{k_{3}+1}+\sum_{j=3}^{q} \frac{1}{k_{j}+1}\right)
$$

It follows from (3.21) and (3.22) that $B_{1}=B_{2}=0$.

This contradicts to (3.16). Hence $f_{1}(z) \equiv f_{2}(z)$. 
As a consequence of Theorem 3.2, we get the following corollary

Corollary 3.2. Let $f_{1}$ and $f_{2}$ be two transcedental or admissible meromorphic functions on the annulus $\mathbb{A}=\left\{z: \frac{1}{R_{0}}<|z|<R_{0}\right\}$, where $1<R<$ $R_{0} \leq+\infty$. Let $a_{j}(j=1,2, \ldots, q)$ be $q$ distinct complex numbers. Then:

(i) If $q=6$ and $\bar{E}_{1)}\left(a_{j}, f_{1}\right)=\bar{E}_{1)}\left(a_{j}, f_{2}\right),(j=1,2, . ., 6)$,

$$
\sum_{j=1}^{6} \max \left\{\Delta_{0}\left(a_{j}, f_{1}\right), \Delta_{0}\left(a_{j}, f_{2}\right)\right\}>0,
$$

then $f_{1}(z) \equiv f_{2}(z)$;

(ii) If $q=5$ and $\bar{E}_{2)}\left(a_{j}, f_{1}\right)=\bar{E}_{2)}\left(a_{j}, f_{2}\right),(j=1,2, . ., 5)$,

$$
\sum_{j=1}^{5} \max \left\{\Delta_{0}\left(a_{j}, f_{1}\right), \Delta_{0}\left(a_{j}, f_{2}\right)\right\}>0
$$

then $f_{1}(z) \equiv f_{2}(z)$.

\section{Acknowledgments}

The second author was supported by the UGC- Rajiv Gandhi National Fellowship (no. F1-17.1/2013-14-SC-KAR-40380) of India.

\section{References}

[1] T.B. Cao, Z.S. Deng, On the uniqueness of meromorphic functions that share three or two finite sets on annuli, it Proceeding Mathematical Sciences, 122 (2012), 203-220.

[2] T.B. Cao, H.X. Yi, Uniqueness theorems of meromorphic functions share sets IM on Annili, Acta Math. Sinica (Chin. Ser.), 54 (2011), 623-632.

[3] T.B. Cao, H.X. Yi, H.Y. Xu, On the multiple values and uniqueness of meromorphic functions on annuli, Compute. Math. Appl., 58 (2009), 1457-1465.

[4] Y.X. Chen, Z.J. Wu, Exceptional values of meromorphic functions and of their derivatives on annuli, Ann. Polon. Math., 105 (2012), 154-165.

[5] A. Fernandez, On the value distribution of meromorphic function in the punctured plane, Mathematychin Studii, 34 (2010), 136-144.

[6] A.Ya. Khrystiyanyn, A.A. Kondratyuk, On the Nevanlinna Theory for meromorphic functions on annuli. I, Mathematychin Studii, 23 (2005), 19-30. 
[7] A.Ya. Khrystiyanyn, A.A. Kondratyuk, On the Nevanlinna Theory for meromorphic functions on annuli. II, Mathematychin Studii, 24 (2005), 57-68.

[8] R. Korhonen, Nevanlinna theory in an annulus, value distribution theory and related topics, Adv Complex Anal. Appl., 3 (2004), 547-554.

[9] S. Axler, Harmonic functions from a complex analysis view point, Amer. Math. Mon., 93 (1986), 246-258.

[10] H.Y. Xu, Z.X. Xuan, The uniqueness of analytic functions on annuli sharing some values, Abstract and Applied analysis, 2012, Article ID 896596, 13 pages (2012).

[11] Hong-Yan Xu, Exceptional Values of Meromorphic Function on Annulus, Hindawi Publishing Corporation The Scientific World Journal Volume 2013,Article ID 937584,7 pages (2013).

[12] H.X. Yi, Notes on a theorem of H. Ueda, J. Shandong Univ., 23, No. 4 (1988), 71-77.

[13] H.X. Yi, C.C. Yang, Uniqueness Theory of Meromorphic Functions. Science Press, Kluwer (2003). 
